\title{
RANGE TRANSFORMATIONS ON A BANACH FUNCTION ALGEBRA
}

\author{
OSAMU HATORI
}

\begin{abstract}
We study the range transformations $\operatorname{Op}\left(A_{D}, \operatorname{Re} B\right)$ and $\operatorname{Op}\left(A_{D}, B\right)$ for Banach function algebras $A$ and $B$. As a special instance, the harmonicity of functions in $\operatorname{Op}\left(A_{D}, \operatorname{Re} A\right)$ for a nontrivial function algebra $A$ is established and is compared with previous investigations of $\mathrm{Op}\left(A_{D}, A\right)$ and $\mathrm{Op}\left((\operatorname{Re} A)_{l},(\operatorname{Re} A)\right)$ for an interval $I$. In $\$ 2$ we present some results on $\mathrm{Op}\left(A_{D}, B\right)$ and use them to show that functions in $\operatorname{Op}^{c}\left(A_{D}, B\right)$ are analytic for certain Banach function algebras.
\end{abstract}

Introduction. We say that $A$ is a Banach function algebra on a compact Hausdorff space $X$ if $A$ is a unital subalgebra of $C(X)$ which is a Banach algebra with the norm $N_{A}(\cdot)$ such that $A$ separates the points of $X$. A function algebra on $X$ is a Banach function algebra on $X$ with the uniform norm as the Banach algebra norm. We denote by $\|\cdot\|_{Y}$ the uniform norm on a subset $Y$ of $X$. If $E$ is a normed space, we denote by $\tilde{E}$ the set of all bounded sequences in $E$. If $A$ is a Banach function algebra, then $\tilde{A}$ is a unital Banach algebra lying in $C(\tilde{X})$, where $\tilde{X}$ is the Stone-Čech compactification of the direct product $X \times N$ of the discrete space $N$ of all positive integers and $X$, with the Banach algebra norm $\tilde{N}_{A}(\tilde{f})=\sup \left\{N_{A}\left(f_{n}\right): n=1,2, \ldots\right\}$ for $\tilde{f}=\left(f_{n}\right)$ in $\tilde{A}$. In this paper we denote Banach function algebras on a compact Hausdorff space $X$ by $A$ or $B$ unless we mention it.

Definition 1 . We say that $A$ is ultraseparating if $\tilde{A}$ separates the points of $\tilde{X}$.

Definition 2. Let $x$ be a point in $X$. We denote:

$$
\begin{gathered}
A_{x}=\{f \in A: f(x)=0\}, \\
Q\left(A_{x}\right)=\left\{\tilde{x} \in \tilde{X}: \tilde{f}(\tilde{x})=0 \forall \tilde{f} \in A_{x}\right\}, \\
F_{x}=\bigcap_{\alpha}\left(G_{\alpha} \times N\right)^{-},
\end{gathered}
$$

where $G_{\alpha}$ varies over all the compact neighborhoods of $x$ and $\left(G_{\alpha} \times N\right)^{-}$is the closure of $G_{\alpha} \times N$ in $\tilde{X}$.

$A_{x}$ is closed in $A$ since $\|\cdot\|_{X} \leqslant N(\cdot)$ for every Banach function algebra $A$ on $X$. We also remark that $\left(G_{\alpha} \times N\right)^{-}$is homeomorphic to $\tilde{G}_{\alpha}$ by the definition of the Stone-Čech compactification. For each $p$ in $\tilde{X}$, the functional $f \mapsto(f)(p)$ on $C(X)$

Received by the editors May 13, 1985 and, in revised form, November 1, 1985.

1980 Mathematics Subject Classification. Primary 46J10: Secondary 46H30.

Key nords and phrases. Function algebra, Banach function algebra, range transformation, ultraseparating.

1986 American Mathematical Society $0002-9947 / 86 \$ 1.00+\$ .25$ per page 
is linear and multiplicative, where $(f)$ denotes the element $(f, f, \ldots)$ in $(C(X))^{\sim}=$ $C(\tilde{X})$, so there is a unique $x_{p}$ in $X$ such that $f\left(x_{p}\right)=(f)(p)$ for all $f$ in $C(X)$.

Lemma 1. We have $F_{x}=\left\{p \in \tilde{X}: x_{p}=x\right\}$ for every $x$ in $X$, and $\tilde{X}=\cup_{x \in X} F_{x}$ is a disjoint union.

ProOf. Let $q$ be in $\left\{p \in \tilde{X}: x_{p}=x\right\} \sim F_{x}$. Then there is a compact neighborhood $G_{\alpha}$ of $x$ such that $q \notin\left(G_{\alpha} \times N\right)^{-}$. So we have $q \in\left(\left(\operatorname{Int} G_{\alpha}\right)^{c} \times N\right)^{-}$since $\tilde{X}=\left(G_{\alpha} \times N\right)^{-} \cup\left(\left(\operatorname{Int} G_{\alpha}\right)^{c} \times N\right)^{-}$, where $\left(\operatorname{Int} G_{\alpha}\right)^{c}$ is the complement of the interior of $G_{\alpha}$ in $X$. Therefore we see that $x=x_{q}$ is in $\left(\operatorname{Int} G_{\alpha}\right)^{c}$ which is a contradiction.

Conversely, let $q$ be in $F_{x}$; then $x_{q}$ is in every compact neighborhood of $x$, so we have $x_{q}=x$. It is trivial that $\tilde{X}=\bigcup F_{x}$ is a disjoint union.

Lemma 2. $Q\left(A_{x}\right) \subset F_{x}$ for every $x$ in $X$.

Proof. Suppose that $p$ is in $Q\left(A_{x}\right) \sim F_{x}$. Then we have $x_{p} \neq x$ by Lemma 1 , so there exists an $f$ in $A_{x}$ such that $f\left(x_{p}\right) \neq f(x)=0$. It follows that $(f)(p)=f\left(x_{p}\right)$ $\neq f(x)=0$, which contradicts $p \in Q\left(A_{x}\right)$.

Lemma 3. $A$ is ultraseparating if and only if $\tilde{A}$ separates the points of $F_{x}$ for every $x$ in $X$.

Proof. Let $p$ and $q$ be different points in $\tilde{X}$. Suppose that $p$ is in $F_{x}$ and $q$ is in $F_{y}$ for different points $x$ and $y$ in $X$, so $x_{p}=x$ and $x_{q}=y$ by Lemma 1 . Since $A$ separates the points of $X$, there is an $f$ in $A$ such that $f(x) \neq f(y)$. We then have

$$
(f)(p)=f\left(x_{p}\right) \neq f\left(x_{q}\right)=(f)(q),
$$

so $\tilde{A}$ separates $p$ and $q$.

LEMMA 4. If $A$ is ultraseparating, then we have

$$
Q\left(A_{x}\right)=(\{x\} \times N)
$$

for every $x$ in $X$.

Proof. $Q\left(A_{x}\right) \supset(\{x\} \times N)^{-}$is trivial by the definition. Suppose that $\tilde{x} \in$ $Q\left(A_{x}\right) \sim(\{x\} \times N)^{-}$. We can choose a finite number of functions $\tilde{f}_{1}=\left(f_{m}^{(1)}\right)$, $\tilde{f}_{2}=\left(f_{m}^{(2)}\right), \ldots, \tilde{f}_{n}=\left(f_{m}^{(n)}\right), \tilde{g}_{1}=\left(g_{m}^{(1)}\right), \tilde{g}_{2}=\left(g_{m}^{(2)}\right), \ldots, \tilde{g}_{n}=\left(g_{m}^{(n)}\right)$ in $\tilde{A}$ such that

$$
\begin{gathered}
\left|\tilde{f}_{1}\right|+\left|\tilde{f}_{2}\right|+\cdots+\left|\tilde{f}_{n}\right|-\left(\left|\tilde{g}_{1}\right|+\left|\tilde{g}_{2}\right|+\cdots+\left|\tilde{g}_{n}\right|\right)>\frac{1}{2} \quad \text { at } \tilde{x}, \\
\left|\tilde{f}_{1}\right|+\left|\tilde{f}_{2}\right|+\cdots+\left|\tilde{f}_{n}\right|-\left(\left|\tilde{g}_{1}\right|+\left|\tilde{g}_{2}\right|+\cdots+\left|\tilde{g}_{n}\right|\right)<-\frac{1}{2} \text { on }(\{x\} \times N)
\end{gathered}
$$

by the Stone-Weierstrass theorem since $\tilde{A}$ separates the points of $\tilde{X}$. Then we have

$$
\tilde{f}_{j}(\tilde{x})=\left(f_{m}^{(j)}(x)\right)(\tilde{x}), \quad \tilde{g}_{j}(\tilde{x})=\left(g_{m}^{(j)}(x)\right)(\tilde{x})
$$


for every $j=1,2, \ldots, n$ because $\tilde{f}_{j}-\left(f_{m}^{(j)}(x)\right)$ and $\tilde{g}_{j}-\left(g_{m}^{(j)}(x)\right)$ are functions in $\tilde{A}_{x}$. So we see that

$$
\begin{aligned}
& \left\{\left|\left(f_{m}^{(1)}(x)\right)\right|+\left|\left(f_{m}^{(2)}(x)\right)\right|+\cdots+\left|\left(f_{m}^{(n)}(x)\right)\right|\right. \\
& \left.\quad-\left|\left(g_{m}^{(1)}(x)\right)\right|+\left|\left(g_{m}^{(2)}(X)\right)\right|-\cdots-\left|\left(g_{m}^{(n)}(x)\right)\right|\right\}(\tilde{x})>\frac{1}{2}
\end{aligned}
$$

and

$$
\begin{aligned}
& \left\{\left|f_{k}^{(1)}(x)\right|+\left|f_{k}^{(2)}(x)\right|+\cdots+\left|f_{k}^{(n)}(x)\right|\right. \\
& \left.\quad-\left|g_{k}^{(1)}(x)\right|-\left|g_{k}^{(2)}(x)\right|-\cdots-\left|g_{k}^{(n)}(x)\right|\right\}<-\frac{1}{2}
\end{aligned}
$$

for every positive integer $k$, since $\tilde{f}_{j}((k, x))=f_{k}^{(j)}(x)$ and $\tilde{g}_{j}((k, x))=g_{k}^{(j)}(x)$ for every $j=1,2, \ldots, n$ and every positive integer $k$. The function

$$
\begin{aligned}
& \left|\left(f_{m}^{(1)}(x)\right)\right|+\left|\left(f_{m}^{(2)}(x)\right)\right|+\cdots+\left|\left(f_{m}^{(n)}(x)\right)\right| \\
& \quad-\left|\left(g_{m}^{(1)}(x)\right)\right|-\left|\left(g_{m}^{(2)}(x)\right)\right|-\cdots-\left|\left(g_{m}^{(n)}(x)\right)\right|
\end{aligned}
$$

in $C(\tilde{X})$ is negative on $\tilde{X}$ by (ii), a contradiction to (i). Therefore we conclude that $Q\left(A_{x}\right) \subset(\{x\} \times N)^{-}$.

Definition 3. Let $E$ and $F$ be subsets of $C(X)$ and let $D$ be a subset of the complex plane $C$. We denote by $\operatorname{Op}\left(E_{D}, F\right)$ the set of all complex-valued functions $h$ on $D$ which satisfy that $h \circ f$ is in $F$ for every $f$ in $E$ with range in $D$. We denote $\operatorname{Op}^{c}\left(E_{D}, F\right)=\operatorname{Op}\left(E_{D}, F\right) \cap C(D)$, where $C(D)$ is the set of all complex-valued continuous functions on $D$.

In [15] de Leeuw and Katznelson investigated the range transformation $\operatorname{Op}\left(A_{D}, A\right)$ for a Banach function algebra $A$. They showed, for example, that $\operatorname{Op}^{c}\left(A_{D}, A\right)=$ $H(D)=\{f \in C(D): f$ is analytic on $D\}$ for a nontrivial function algebra $A$ and the open unit disk $D$. Later Spraglin [17] removed the continuity of the operating function for the above result (cf. $[1,2,10])$. He in fact proved that $\operatorname{Op}\left(A_{Y}, A\right)=$ $\mathrm{Op}^{c}\left(A_{Y}, A\right)$ for a function algebra on an infinite compact Hausdorff space and for a plane domain $Y$. Bernard and Dufresnoy showed $\bar{z} \notin \mathrm{Op}\left(A_{D}, A\right)$ for the case that $A$ is a nontrivial ultraseparating Banach function algebra. On the other hand, functions in $\mathrm{Op}\left((\operatorname{Re} A)_{l}, \operatorname{Re} A\right)$ for an interval $I$ were first studied by $\mathrm{J}$. Wermer [18]. In [11 and 16] it was proved that the functions in $\left.\operatorname{Op}\left((\operatorname{Re} A)_{I}, \operatorname{Re} A\right)\right)$ are affine on $I$ for a nontrivial function algebra and an analogous result for a nontrivial ultraseparating Banach function algebra is given in [12]. The question of whether functions in $\mathrm{Op}\left(A_{Y}, \operatorname{Re} A\right)$ are harmonic for a function algebra $A$ naturally follows from these theorems. In $\S 1$ we obtain a result on $\operatorname{Op}\left(A_{Y}, \operatorname{Re} B\right)$ and we show, as a special instance, an affirmative answer to the question. The main emphasis in $\S 2$ will be on $\mathrm{Op}\left(A_{Y}, B\right)$. We show that $\operatorname{Op}^{c}\left(A_{D}, B\right)=H(D)$ if and only if $A \subset B \neq C(X)$ in the case that $B$ is a function algebra on $X$ or $A$ is an ultraseparating Banach function algebra on $X$, which provides information on the question in [2]. We will also generalize a theorem of Bernard and Dufresnoy (cf. [6]). 
1. Functions in $\mathrm{Op}\left(A_{D}, \operatorname{Re} B\right)$. In this section we study functions in a certain range transformation $\operatorname{Op}\left(A_{D}, \operatorname{Re} B\right)$.

Let $h$ be a complex-valued continuous function on the closed unit disk $\bar{D}=\{z \in$ $C:|z| \leqslant 1\}$ and $\Delta_{\delta}(w)$ a smoothing operator of class $C^{\infty}$ supported in $D_{\delta}=\{z \in C$ : $|z|<\delta\}$, that is, $\Delta_{\delta}(w)$ is a nonnegative real-valued $C^{\infty}$ function on the complex plane $C$ supported in $D_{\delta}$ with integral 1. For $0<\delta<\frac{1}{2}$, for $\left(z_{1}, z_{2}\right) \in \bar{D}_{1-\delta} \times \bar{D}=$ $\left\{\left(z_{1}, z_{2}\right) \in C^{2}:\left|z_{1}\right| \leqslant 1-\delta,\left|z_{2}\right| \leqslant 1\right\}$, and for $\alpha \in C$, put

$$
\begin{aligned}
& \quad h_{\delta}\left(z_{1}, z_{2}\right)=\iint h\left(z_{1}-z_{2} w\right) \Delta_{\delta}(w) d x d y, \\
& H_{\delta}\left(z_{1}, z_{2}, \alpha\right) \\
& \quad=\iint h\left(z_{1}-z_{2} w\right)\left\{\left(\operatorname{Re} \alpha z_{2}\right) \partial / \partial x \Delta_{\delta}(w)+\left(\operatorname{Im} \alpha z_{2}\right) \partial / \partial y \Delta_{\delta}(w)\right\} d x d y, \\
& L_{\delta}\left(z_{1}, z_{2}, \alpha\right)=\iint h\left(z_{1}-z_{2} w\right)\left|\alpha z_{2}\right|^{2} \Delta\left(\Delta_{\delta}(w)\right) d x d y,
\end{aligned}
$$

where $\Delta$ denotes the Laplacian with respect to $x=\operatorname{Re} w$ and $y=\operatorname{Im} w$.

LemMa 5. Let $\Delta_{1}$ denote the Laplacian with respect to $\operatorname{Re} z_{1}$ and $\operatorname{Im} z_{1}$. If $0<\delta<\frac{1}{2}$ and $M>0$, then

$$
\begin{aligned}
|\alpha|^{2} \Delta_{1}\left(h_{\delta}\left(z_{1}, z_{2}\right)\left|z_{2}\right|^{4}\right) & = \begin{cases}\left|\alpha z_{2}^{2}\right|^{2} \Delta_{1}\left(h_{\delta}\left(z_{1}, z_{2}\right)\right) & \text { if } z_{2} \neq 0, \\
0 & \text { if } z_{2}=0\end{cases} \\
& =L_{\delta}\left(z_{1}, z_{2}, \alpha\right)
\end{aligned}
$$

and the restriction of this function to $\bar{D}_{1-2 \delta} \times \bar{D} \times \bar{D}_{M}=\left\{\left(z_{1}, z_{2}, \alpha\right) \in C^{3}:\left|z_{1}\right| \leqslant 1\right.$ $\left.-2 \delta,\left|z_{2}\right| \leqslant 1,|\alpha| \leqslant M\right\}$ lies, for each $\gamma$ such that $0<\gamma<1 /(2 M)$, in $I_{\delta, M, \gamma}$, the uniformly closed subspace of $C\left(\bar{D}_{1-2 \delta} \times \bar{D} \times \bar{D}_{M}\right)$ generated by $\left\{h_{\delta}\left(z_{1}-\beta \alpha z_{2}^{2}, z_{2}\right)\right.$ : $\beta \in C,|\beta|<\delta \gamma\}$.

PROOF. The equality is proven by direct computation, using difference quotients.

As the small real number $t$ tends to 0 we have

uniformly on $\bar{D}_{1-1.5 \delta} \times \bar{D} \times \bar{D}_{M}$, and

$$
\left\{h_{\delta}\left(z_{1}+\alpha z_{2}^{2} t, z_{2}\right)-h_{\delta}\left(z_{1}, z_{2}\right)\right\} / t \rightarrow H_{\delta}\left(z_{1}, z_{2}, \alpha\right)
$$

$$
\begin{aligned}
& \left\{H_{\delta}\left(z_{1}+\alpha z_{2}^{2} t, z_{2}, \alpha\right)-H_{\delta}\left(z_{1}, z_{2}, \alpha\right)\right\} / t \\
& \quad+\left\{H_{\delta}\left(z_{1}+i \alpha z_{2}^{2} t, z_{2}, i \alpha\right)-H_{\delta}\left(z_{1}, z_{2}, i \alpha\right)\right\} / t \rightarrow L_{\delta}\left(z_{1}, z_{2}, \alpha\right)
\end{aligned}
$$

uniformly on $\bar{D}_{1-2 \delta} \times \bar{D} \times \bar{D}_{M}$.

It follows successively that $H_{\delta}\left(z_{1}, z_{2}, \alpha\right), H_{\delta}\left(z_{1}, z_{2}, i \alpha\right)$, and $L_{\delta}\left(z_{1}, z_{2}, \alpha\right)$ belong to $L_{\delta, M, \gamma}$, completing the proof.

The differential operators $\partial / \partial w, \partial / \partial \bar{w}(w=x+i y), \partial / \partial z_{1}, \partial / \partial \bar{z}_{1}\left(z_{1}=x_{1}+i y_{1}\right)$ are defined by

$$
\begin{gathered}
\partial / \partial w=\frac{1}{2}(\partial / \partial x-i \partial / \partial y), \quad \partial / \partial \bar{w}=\frac{1}{2}(\partial / \partial x+i \partial / \partial y), \\
\partial / \partial z_{1}=\frac{1}{2}\left(\partial / \partial x_{1}-i \partial / \partial y_{1}\right), \quad \text { and } \partial / \partial \bar{z}_{1}=\frac{1}{2}\left(\partial / \partial x_{1}+i \partial / \partial y_{1}\right) .
\end{gathered}
$$

LEMMA 6. If $0<\delta<\frac{1}{2}$ and $M>0$, for $0<\gamma<1 /(2 M)$ the functions

$$
\alpha \partial / \partial z_{1}\left(h_{\delta}\left(z_{1}, z_{2}\right) z_{2}^{2}\right)= \begin{cases}\alpha z_{2}^{2} \partial / \partial z_{1}\left(h_{\delta}\left(z_{1}, z_{2}\right)\right) & \text { if } z_{2} \neq 0 \\ 0 & \text { if } z_{2}=0\end{cases}
$$


and

$$
\bar{\alpha} \partial / \partial \bar{z}_{1}\left(h_{\delta}\left(z_{1}, z_{2}\right) \bar{z}_{2}^{2}\right)= \begin{cases}\bar{\alpha} \bar{z}_{2}^{2} \partial / \partial \bar{z}_{1}\left(h_{\delta}\left(z_{1}, z_{2}\right)\right) & \text { if } z_{2} \neq 0, \\ 0 & \text { if } z_{2}=0\end{cases}
$$

restricted to $\bar{D}_{1-2 \delta} \times \bar{D} \times \bar{D}_{M}$ belong to $I_{\delta, M, \gamma}$.

Proof. Again by direct computation,

$$
\begin{aligned}
\alpha \partial / \partial z_{1}\left(h_{\delta}\left(z_{1}, z_{2}\right) z_{2}^{2}\right) & =\iint h\left(z_{1}-z_{2} w\right) \alpha z_{2} \partial / \partial w \Delta_{\delta}(w) d x d y \\
& =\frac{1}{2} H_{\delta}\left(z_{1}, z_{2}, \alpha\right)-\frac{i}{2} H_{\delta}\left(z_{1}, z_{2}, i \alpha\right)
\end{aligned}
$$

and

$$
\begin{aligned}
\bar{\alpha} \partial / \partial \bar{z}_{1}\left(h_{\delta}\left(z_{1}, z_{2}\right) \bar{z}_{2}^{2}\right) & =\iint h\left(z_{1}-z_{2} w\right) \bar{\alpha} \bar{z}_{2} \partial / \partial \bar{w} \Delta_{\delta}(w) d x d y \\
& =\frac{1}{2} H_{\delta}\left(z_{1}, z_{2}, \alpha\right)+\frac{i}{2} H_{\delta}\left(z_{1}, z_{2}, i \alpha\right) .
\end{aligned}
$$

As was noted during the proof of Lemma $5, H_{\delta}\left(z_{1}, z_{2}, \alpha\right)$ and $H_{\delta}\left(z_{1}, z_{2}, i \alpha\right)$ belong to $I_{\delta, M, \gamma}$.

We will use Lemmas 5 and 6 to prove the main results in $\S \S 1,2$.

For a subset $E$ of $C(X)$, we denote by $\mathrm{cl} E$ the uniform closure of $E$ in $C(X)$.

THEOREM 1. Suppose that $A$ and $B$ are Banach function algebras on $X$ and that $D$ is a plane domain. We assume the following condition:

For every pair of points $\tilde{x}$ and $\tilde{y}$ in $\tilde{X}, \tilde{A}$ separates them whenever $(\mathrm{cl} B)^{\sim}$ separates them.

If $B \neq C(X)$, then every function in $\operatorname{Op}^{c}\left(A_{D}, \operatorname{Re} B\right)$ is harmonic.

REMARK 1. The condition (*) is necessary. An example is the following: Let $X$ be an annulus $\{z \in C: 1 \leqslant|z| \leqslant 2\}$ and let $\Gamma$ be the unit circle $\{z \in C:|z|=1\}$. Suppose that $A=\{f \in C(X): f$ is uniformly approximated by analytic polynomials on $X\}$ and $B=\{f \in C(X): f \mid \Gamma$ is uniformly approximated by analytic polynomials on $\Gamma$ \}. Then $A$ and $B$ are nontrivial function algebras on $X$ and the function $z \mapsto(\operatorname{Re} z)^{2}$ is in $\mathrm{Op}\left(A_{D}, \operatorname{Re} B\right)$ for every plane domain $D$ since every function $\operatorname{Re}(A \mid \Gamma)$ has absolute converging Fourier series.

An example in which condition (*) can be verified while $A$ is not ultraseparating is the following: Let $H^{\infty}$ be the Hardy class of boundary values of bounded analytic functions on the open unit disk and $B$ be a Douglas algebra on the unit circle. We may consider that $H^{\infty}$ and $B$ are function algebras on the maximal ideal space $M(B)$ of $B$. If $\tilde{B}$ separates $\tilde{x}$ and $\tilde{y}$ in $(M(B))^{\infty}$, then $\left(H^{\infty}\right)^{-}$separates them. If $M(B)$ contains a nontrivial Gleason part (for example, $M\left(H^{\infty}+C\right)$ contains one), then $H^{\infty}$ is not ultraseparating. Suppose that $K$ is a closed restriction set for $B$. Then $\left(H^{\infty} \mid K\right)^{-}$separates the points of $(M(B))^{-}$which are separated by $\left(\operatorname{cl}\left(H^{\infty} \mid K\right)\right)^{\sim}[13]$.

REMARK 2. In the same way as in Corollary II.4.1 in [10] we see the following:

Suppose that $F$ is a complex-valued function on a subset $E$ of $C$. Let $f$ be a complex-valued continuous function on a compact Hausdorff space with $f(X) \subset E$. If $F \circ f$ is continuous on $X$, then we see that $F$ is continuous on $f(X)$. Now in the 
case where $A$ is a function algebra on an infinite compact Hausdorff space $X$ we see that $A$ partially interpolates by [10, Theorem II.9]. Thus we have the following:

(a) $\mathrm{Op}\left(A_{D}, B\right)=\mathrm{Op}^{c}\left(A_{D}, B\right)$,

(b) $\mathrm{Op}\left(A_{D}, \operatorname{Re} B\right)=\operatorname{Op}^{c}\left(A_{D}, \operatorname{Re} B\right)$,

(c) $\left.\left.\mathrm{Op}\left((\operatorname{Re} A)_{I}, \operatorname{Re} B\right)\right)=\mathrm{Op}^{c}\left((\operatorname{Re} A)_{I}, \operatorname{Re} B\right)\right)$,

where $D$ is a plane domain and $I$ is a nondegenerate interval. On the other hand, every Banach function algebra on a finite $X$ is the trivial one.

Proof of Theorem 1. Süpose that an $h \in \operatorname{Op}^{c}\left(A_{D}, \operatorname{Re} B\right)$ is not harmonic. Without loss of generality we may assume that $D=\{z \in C:|z|<1\}, h$ is continuous on $\bar{D}=\{z \in C:|z| \leqslant 1\}, h$ is not harmonic at the origin, and $h(0)=0$. Let $x$ be a point in $X$.

LEMMA 1.1. cl $B$ is ultraseparating.

Proof. Put

$$
h_{\delta}(z)=h_{\delta}(z, 1)=\iint h(z-w) \Delta_{\delta}(w) d x d y \quad(w=x+i y)
$$

for a small $\delta>0$ and a smoothing operator $\Delta_{\delta}(w)$ of class $C^{\infty}$ supported in $\{z \in C:|z|<\delta\}$. Then $h_{\delta}(z)$ is of class $C^{\infty}$ on $\{z \in C:|z| \leqslant 1-2 \delta\}$ and $h_{\delta}$ tends to $h$ as $\delta$ tends to 0 . So for every $\varepsilon>0$ there is $\delta(\varepsilon)>0$ such that $\Delta\left(h_{\delta(\varepsilon)}\left(z_{\varepsilon}\right)\right) \neq 0$ for some $z_{\varepsilon}$ with $\left|z_{\varepsilon}\right|<\min \{\varepsilon, 1-2 \delta(\varepsilon)\}$. For every $f$ in $A$, we have

$$
|f|^{2} \Delta_{1}\left(h_{\delta(\varepsilon)}\left(z_{\varepsilon}, 1\right) 1^{4}\right) \in \operatorname{cl}(\operatorname{Re} B)=|f|^{2} \Delta_{1}\left(h_{\delta(\varepsilon)}\left(z_{\varepsilon}\right)\right)
$$

by Lemma 5. Thus we see that $|f|^{2} \in \operatorname{cl}(\operatorname{Re} B)$ for every $f$ in $A$. It follows that $\operatorname{cl}(\operatorname{Re} B)=C_{R}(X)$ by the Stone-Weierstrass theorem so that $\mathrm{cl} B$ is a Dirichlet algebra. Then $\operatorname{cl} B$ is ultraseparating.

We can prove the following boundedness principle for $h$ in the same way as in the proof of the Theorem in [12].

LemMA 1.2. For each $x$ and $X$ there are a positive integer $n_{0}$, a real number $\varepsilon$, $0<\varepsilon<\frac{1}{2}$, and an $F$ in $\left\{f \in A_{x}:\|f\|_{X} \leqslant \frac{1}{2}\right\}$ such that there is a dense subset $U$ of $\left\{g \in A_{x}: N_{A}(g-F)<\varepsilon\right\}$ with $F$ in $U$ which satisfy the following:

For every $g$ in $U$ we have $\|g\|_{X} \leqslant \frac{1}{2}$ and

$$
N_{\operatorname{Re} B}(h \circ g)<n_{0} \text {, }
$$

where $N_{\operatorname{Re} B}(u)=\inf \left\{N_{B}(f): f \in B\right.$ and $\left.\operatorname{Re} f=u\right\}$ for the norm $N_{B}(\cdot)$ on $B$.

Proof of Theorem 1 (SEQuel). By Lemma 1.1 and condition (*) we see that $A$ is ultraseparating. Thus there is a positive number $M$ such that for every pair of different points $x$ and $y$ in $X$, there is a function $f$ in $A$ with $f(x)=0, f(y)=1$, and $N_{A}(f)<M$ [5]. Given $x$ in $X$, take $z_{2}$ in $C$ with $0<\left|z_{2}\right|<\varepsilon /(2 M)$ with $F, \varepsilon$, $U$ as in Lemma 1.2. In the same way as in the proof of Lemma 1.1, for every $\delta$, $0<\delta<\min \left\{\frac{1}{2}, M / \varepsilon\right\}$, and every $\eta, 0<\eta<\min \left\{\delta\left|z_{2}\right|, 1-2 \delta\right\}$, there is a $\delta(0)$ with $0<\delta(0)<\delta$ such that $h_{\delta(0)}\left(z, z_{2}\right)$ is not harmonic on $\{z \in C:|z|<\eta\}$. Thus we see that there is a $z_{0}$ with $\left|z_{0}\right|<\eta$ such that

$$
\Delta_{1}\left(h_{\delta(0)}\left(z_{0}, z_{2}\right)\left|z_{2}\right|^{4}\right) \neq 0 \text {. }
$$


By Lemma 5 we have

$$
\Delta_{1}\left(h_{\delta(0)}\left(z_{0}, z_{2}\right)\left|z_{2}\right|^{4}\right)=\iint h\left(z_{0}-z_{2} w\right)\left|z_{2}\right|^{2} \Delta\left(\Delta_{\delta(0)}(w)\right) d x d y,
$$

and changing the variable of integration by $w^{\prime}=w-z_{0} / z_{2}$ we have

$$
=\iint h\left(-z_{2} w^{\prime}\right)\left|z_{2}\right|^{2} \Delta\left(\Delta_{\delta(0)}\left(w^{\prime}+z_{0} / z_{2}\right)\right) d x d y,
$$

where $\Delta_{\delta(0)}\left(w^{\prime}+z_{0} / z_{2}\right)$ is a smoothing operator of class $C^{\infty}$ supported in $\{z \in C$ : $|z|<2 \delta\}$. Thus without loss of generality we may assume that $z_{0}=0$. So we conclude that $h_{\delta(0)}(z, w)|w|^{4}$ is of class $C^{\infty}$ with respect to $\operatorname{Re} z$ and $\operatorname{Im} z$ and

$$
\left|\Delta_{1}\left(h_{\delta(0)}(z, w)|w|^{4}\right)\right| \geqslant \frac{1}{2}\left|\Delta_{1}\left(h_{\delta(0)}\left(0, z_{2}\right)\left|z_{2}\right|^{4}\right)\right| \neq 0
$$

on $\left\{(z, w) \in C^{2}:|z| \leqslant \varepsilon^{\prime},\left|w-z_{2}\right| \leqslant \varepsilon^{\prime}\right\}$ for some $\varepsilon^{\prime}>0$.

Put $K_{x}=\left\{a \in X:|F(a)|<\varepsilon^{\prime} / 2\right\}$ and let $y \in K_{x} \sim\{x\}$. There is an $\alpha>0$ with $|F(y)|<\varepsilon^{\prime} / 2-\alpha$ and $g$ in $A_{x}$ such that $g(y)=1$ and $N_{A}(g) \leqslant M$. So

$$
G_{y}=\left\{b \in K_{x}:|F(b)| \leqslant \varepsilon^{\prime} / 2-\alpha,|1-g(b)|<\min \left\{\varepsilon^{\prime} /\left(2\left|z_{2}\right|\right), 1 / 2\right\}\right\}
$$

is a compact neighborhood of $y$ such that $x \notin G_{y} \subset K_{x}$. For every $\tilde{F}$ in $\tilde{A}$ and complex numbers $\beta$ and $w$ with sufficiently small absolute values $|\beta|$ and $|w|<\delta(0)$ we have

$$
h\left((F)+\tilde{F}\left(z_{2} g\right)^{2} \beta-\left(z_{2} g\right) w\right) \in \operatorname{cl} \operatorname{Re} \tilde{B}
$$

by Lemma 1.2 since we see

$$
\tilde{N}\left(\tilde{F}\left(z_{2} g\right)^{2} \beta-\left(z_{2} g\right) w\right)<\varepsilon^{2}|\beta| \tilde{N}(\tilde{F})+\varepsilon \delta(0)<\varepsilon
$$

for a $w$ and a $\beta$ with sufficiently small absolute values. It follows that

$$
|\tilde{F}|^{2} \Delta_{1}\left(h_{\delta(0)}\left((F),\left(z_{2}\right)\right)\left|\left(z_{2} g\right)\right|^{4}\right)
$$

is in $\mathrm{cl} \operatorname{Re} \tilde{B}$ by Lemma 5 since

$$
h_{\delta(0)}\left((F)+\beta \tilde{F}\left(z_{2} g\right)^{2},\left(z_{2} g\right)\right)
$$

is in $\operatorname{cl} \operatorname{Re} \tilde{B}$. We see that $|(F)|<\varepsilon^{\prime} / 2$ and $\left|z_{2}-\left(z_{2} g\right)\right| \leqslant \varepsilon^{\prime} / 2$ on $\tilde{G}_{y}$ by the definition of $\tilde{G}_{y}$, so the inequality

$$
\left|\Delta_{1}\left(h_{\delta(0)}\left((F),\left(z_{2} g\right)\right)\left|\left(z_{2} g\right)\right|^{4}\right) \geqslant \frac{1}{2}\right| \Delta_{1}\left(h_{\delta(0)}\left(0, z_{2}\right)\left|z_{2}\right|^{4}\right) \neq 0
$$

holds on $\tilde{G}_{y}$. Since $\tilde{A}$ separates the points of $\tilde{X}$, we have

$$
C_{R}\left(\tilde{G}_{y}\right) \subset \operatorname{cl}\left(\operatorname{cl} \operatorname{Re} \tilde{B} \mid \tilde{G}_{y}\right)=\operatorname{cl}\left(\operatorname{Re} \tilde{B} \mid \tilde{G}_{y}\right)=\operatorname{cl}\left(\operatorname{Re} B \mid G_{y}\right)^{\sim}
$$

by the Stone-Weierstrass theorem. It follows by Bernard's lemma [5] that $\operatorname{Re} B \mid G_{y}=$ $C_{R}\left(G_{y}\right)$. Thus we see that $B \mid G_{y}=C\left(G_{y}\right)$ by a theorem of Bernard on Banach function algebras with uniformly closed real parts [5]. By compactness of $X$ we have a finite number of points $x(1), x(2), \ldots, x(n)$ in $X$ such that the open neighborhoods $K_{x(1)}, K_{x(2)}, \ldots, K_{x(n)}$ of the points $x(j)$ cover $X$. Thus we conclude the following:

For every $y$ in $X \sim\{x(1), x(2), \ldots, x(n)\}$, there is a compact neighborhood $G_{y}$ with $G_{y} \subset K_{x(j)}$ for some $j=1,2, \ldots, n$ such that $B \mid G_{y}=C\left(G_{y}\right)$. If $B$ is a function algebra we have the conclusion $B=C(X)$.

To prove the general case we need some lemmas. 
Lemma 1.3. For every $x$ in $X, F_{x}$ is a p-set for $\mathrm{cl} \tilde{B}$, that is, $F_{x}$ is an intersection of peak sets for $\mathrm{cl} \tilde{B}$.

Proof. Every compact neighborhood of $x$ is a $p$-set for $\operatorname{cl} B$ since we have cl $B=C(X)$ by the above argument. If $G_{\alpha}$ is a compact neighborhood of $x$, there exist a compact neighborhood $G_{\beta}$ of $x$ and a peak set $Y_{\alpha}$ for $C(X)$ such that $G_{\beta} \subset Y_{\alpha} \subset$ Int $G_{\alpha}$. Put $\check{Y}_{\alpha}=\left\{p \in \tilde{X}:\left(f_{\alpha}\right)(p)=1\right\}$, where $f_{\alpha} \in C(X)$ is a peaking function for $Y_{\alpha}$, so $\check{Y}_{\alpha}$ is a peak set for $\mathrm{cl} \tilde{B}$ and we have

$$
\left(G_{\beta} \times N\right)^{-} \subset \check{Y}_{\alpha} \subset\left(G_{\alpha} \times N\right)^{-},
$$

which proves that $F_{x}=\bigcap_{\alpha} \check{Y}_{\alpha}$ is a $p$-set for $\mathrm{cl} \tilde{B}$.

By Lemma 1.3 we see that $F_{x}$ is a closed restriction set for $\mathrm{cl} \tilde{B}_{x}$.

Lemma 1.4. Suppose that $Y$ is an antisymmetric set for $\mathrm{cl} \tilde{B}$. Then there is an $x$ in $X$ such that $F_{x} \supset Y$. In particular, every maximal antisymmetric set is always contained in some $F_{x}$.

Proof. By Lemma 1 we can choose an $x$ in $X$ such that $\exists \tilde{x} \in Y \cap F_{x}$. If we assume $Y \sim F_{x} \neq \varnothing$, then we have a $y$ in $X \sim\{x\}$ such that $\exists \tilde{y} \in Y \cap F_{y}$. Since $\operatorname{cl} B=C(X)$, there exists a real-valued $f$ in $\operatorname{cl} B$ such that $f(x) \neq f(y)$. It follows that $(f)(\tilde{x})=f(x) \neq f(y)=(f)(\tilde{y})$ by Lemma 1. This is a contradiction, for $(f)$ is in cl $\tilde{B}$ by simple calculation and $(f)$ is a nonconstant real-valued function on $Y$. So we have $Y \sim F_{x}=\varnothing$ or $F_{x} \supset Y$.

\section{LEMMA 1.5. $B$ is ultraseparating.}

Proof. By Lemma 3 we need only prove that $\tilde{B}$ separates the points of $F_{x}$ for every $x$ in $X$. There are two cases to consider: (1) $\tilde{a} \in F_{x} \sim Q\left(B_{x}\right)$ and $\tilde{b} \in F_{x} ;$ (2) $\tilde{a}, \tilde{b} \in Q\left(B_{x}\right)$. Note that $Q\left(B_{x}\right) \supset Q\left(A_{x}\right)=(\{x\} \times N)^{-}$by Lemma 4 .

(1) An $\tilde{f}$ in $\tilde{A}$ separates $\tilde{a}$ and $\tilde{b}$ since $A$ is ultraseparating. There is a $\tilde{g}$ in $\tilde{A}_{x}$ such that $\tilde{g}(\tilde{a}) \neq 0$ since $\tilde{a} \notin Q\left(B_{x}\right)$. Thus $\tilde{g}$ and $\tilde{f} \tilde{g}$ are in $\tilde{A}_{x}$ and $\tilde{g}$ or $\tilde{f} \tilde{g}$ separates $\tilde{a}$ and $\tilde{b}$. Suppose that $\tilde{g}_{0} \in \tilde{A}_{x}$ separates $\tilde{a}$ and $\tilde{b}$ and $\tilde{g}_{0}(\tilde{a}) \neq 0$. Put $\tilde{g}_{1}=\tilde{g}_{0}^{2}-$ $\tilde{g}_{0}(\tilde{b}) \tilde{g}_{0}$; so we have $\tilde{g}_{1} \in \tilde{A}_{x}$ and $\tilde{g}_{1}(\tilde{b})=0$, but $\tilde{g}_{1}(\tilde{a}) \neq 0$. Since $h$ is not harmonic at $0, h$ is not constant near the origin. For an appropriate complex number $\alpha$, we have $h\left((F)+\alpha \tilde{g}_{1}\right) \in \operatorname{cl} \operatorname{Re} \tilde{B}$ by Lemma 1.2 and we see that

$$
h\left((F)+\alpha \tilde{g}_{1}\right)(\tilde{a})=h\left(\alpha \tilde{g}_{1}\right)(\tilde{a}) \neq 0=h\left(\alpha \tilde{g}_{1}\right)(\tilde{b})=h\left((F)+\alpha \tilde{g}_{1}\right)(\tilde{b})
$$

since $(F)=0$ on $F_{x}$.

(2) Now $A_{x}$ cannot separate $\tilde{a}$ and $\tilde{b}$ but $\tilde{A}=\tilde{A}_{x}+l^{\infty}$ does because $A$ is ultraseparating; here $l^{\infty}=\left\{\left(c_{n}\right): c_{n}\right.$ is a constant function for each $n$, and $\sup \left|c_{n}\right|<$ $\infty$ ). Thus $l^{\infty}$ must separate $\tilde{a}$ and $\tilde{b}$, hence $\tilde{B}$ separates them since $\tilde{B}$ contains $l^{\infty}$.

By Lemma $1.5 \mathrm{cl} \tilde{B}$ is a function algebra on $\tilde{X}$. If we see that $\operatorname{cl} \tilde{B} \mid F_{x}=C\left(F_{x}\right)$ for every $x$ in $X$, then $\operatorname{cl} \tilde{B}=C(\tilde{X})$ by Lemma 1.4 and Bishop's generalization of the Stone-Weierstrass theorem $[8]$.

Proof OF THeorem 1 (SEQuel). Let ${ }_{0} F_{x}$ be the quotient space of $F_{x}$ for $\tilde{B}_{x}$, that is, the space defined by identifying the points of $F_{x}$ which cannot be separated by $\tilde{B}_{x}$. If $\tilde{x}$ is in $F_{x} \sim Q\left(B_{x}\right)$ and $\tilde{y}$ is in $F_{x}$, then it is easy to see that $\tilde{B}_{x}$ separates $\tilde{x}$ 
and $\tilde{y}$. If $\tilde{x}$ and $\tilde{y}$ are points in $Q\left(B_{x}\right)$, then $\tilde{B}_{x}$ cannot separate them. Thus $Q\left(B_{x}\right)$ is the only subset of $F_{x}$ with more than one element which corresponds to a point in ${ }_{0} F_{x}$. Let $p$ be the point in ${ }_{0} F_{x}$ which corresponds to $Q\left(B_{x}\right)$. Let $\left[\tilde{B}_{x}\right]$ be the uniformly closed algebra generated by $\tilde{B}_{x} \mid F_{x}$ and the constant functions. Then we may view $\left[\tilde{B}_{x}\right]$ as a function algebra on ${ }_{0} F_{x}$. Let $\tilde{x}$ be a point in ${ }_{0} F_{x} \sim\{p\}$. Since $Q\left(B_{x}\right)=Q\left(A_{x}\right)=(\{x\} \times N)^{-}$, there is an $\tilde{f}_{2}$ in $\tilde{A}_{x}$ with $\tilde{N}_{A}\left(\tilde{f}_{2}\right) \leqslant \frac{1}{2}$ such that $\tilde{f}_{2}(\tilde{x})=z_{2} \neq 0$. Take $\delta>0$ with $\tilde{N}_{A}\left(\tilde{f}_{2}\right) \cdot \delta<\varepsilon / 2$, where $\varepsilon$ is the constant in Lemma 1.2. We can take $\delta(0)$ with $0<\delta(0)<\delta$ and a smoothing operator $\Delta_{\delta(0)}(w)$ of class $C^{\infty}$ such that

$$
\Delta_{1}\left(h_{\delta(0)}\left(0, z_{2}\right)\right) \neq 0 .
$$

We can choose $\varepsilon^{\prime}>0$ such that

$$
\left|\Delta_{1}\left(h_{\delta(0)}(z, w)\right)\right| \geqslant \frac{1}{2}\left|\Delta_{1}\left(h_{\delta(0)}\left(0, z_{2}\right)\right)\right|
$$

on $\left\{(z, w) \in C^{2}:|z| \leqslant \varepsilon^{\prime},\left|w-z_{2}\right| \leqslant \varepsilon^{\prime}\right\}$. Put

$$
Y=\left\{\tilde{y} \in{ }_{0} F_{x}:\left|\tilde{f}_{2}(\tilde{y})-\tilde{f}_{2}(\tilde{x})\right| \leqslant \min \left\{\varepsilon^{\prime} / 2,\left|z_{2}\right| / 2\right\}\right\} .
$$

Then $Y$ is a compact neighborhood of $\tilde{x}$ in ${ }_{0} F_{x}$ and we may view $Y$ as a compact set in $F_{x}$ since $Y \nexists p$. For a function $\tilde{F}$ in $\tilde{A}=(\tilde{A})^{\sim}$ take a complex number $\beta$ with sufficiently small absolute value. Then we have

$$
\tilde{N}_{A}\left(\tilde{F}\left(\tilde{f}_{2}\right)^{2} \beta-\left(\tilde{f}_{2}\right) w\right) \leqslant \tilde{N}(\tilde{F}) \tilde{N}\left(\tilde{f}_{2}\right)^{2}|\beta|+\tilde{N}\left(\tilde{f}_{2}\right) \cdot \delta(0)<\varepsilon
$$

for a complex number $w$ with $|w|<\delta(0)$, where we denote $\left(\tilde{f}_{2}\right)=\left(\tilde{f}_{2}, \tilde{f}_{2}, \ldots\right)$ in $\tilde{A}$. So we see by Lemma 1.2 that

$$
h\left(((F))+\tilde{F}\left(\tilde{f}_{2}\right)^{2}-\left(\tilde{f}_{2}\right) w\right) \in \operatorname{cl} \operatorname{Re} \tilde{B}_{x}
$$

since $h(0)=0$. So

$$
h_{\delta(0)}\left(((F))+\tilde{F}\left(\tilde{f}_{2}\right)^{2}-\left(\tilde{f}_{2}\right) w,\left(\tilde{f}_{2}\right)\right) \in \operatorname{cl} \operatorname{Re} \tilde{B}_{x} .
$$

By Lemma 5 we have

$$
|\tilde{F}|^{2} \Delta_{1}\left(h_{\delta(0)}\left(((F)),\left(\tilde{f}_{2}\right)\right)\left|\left(f_{2}\right)^{4}\right|\right) \in \operatorname{cl} \operatorname{Re} \tilde{B}_{x}
$$

so

$$
|\tilde{F}|^{2} \Delta_{1}\left(h_{\delta(0)}\left(0,\left(\tilde{f}_{2}\right)\right)\left|\left(\tilde{f}_{2}\right)^{4}\right|\right)\left|\tilde{Y} \in\left(\operatorname{cl} \operatorname{Re} \tilde{B}_{x}\right)\right| \tilde{Y} \subset \operatorname{cl}\left(\operatorname{Re}\left(\tilde{B}_{x} \mid Y\right)^{\sim}\right)
$$

since $(F)=0$ on $F_{x}$. Thus by the Stone-Weierstrass theorem we have

$$
C_{R}(\tilde{Y}) \subset \operatorname{cl}\left(\operatorname{Re}\left(\tilde{B}_{x} \mid Y\right)^{\sim}\right),
$$

for $\left|\Delta_{1}\left(h_{\delta(0)}\left(0,\left(\tilde{f}_{2}\right)\right)\left|\left(\tilde{f}_{2}\right)^{4}\right|\right)\right|>0$ on $\tilde{Y}$ since $\left|\tilde{f}_{2}-z_{2}\right| \leqslant \varepsilon^{\prime} / 2$ on $Y$. So we have $C_{R}(Y)=\operatorname{Re} \tilde{B}_{x} \mid Y$ by Bernard's lemma and $C(Y)=\left[\tilde{B}_{x}\right] \mid Y$ by a theorem of Bernard. We have just shown that for every point $\tilde{x}$ in ${ }_{0} F_{x} \sim\{p\}$, there is an interpolating compact neighborhood $Y$ of $\tilde{x}$. Thus by [9, Corollary 2.13] we have

$$
\left[\tilde{B}_{x}\right]=C\left({ }_{0} F_{x}\right) \text {. }
$$


Thus $\left(\left[\tilde{B}_{x}\right]+l^{\infty}\right) \mid F_{x}$ is a selfadjoint subalgebra of $C\left(F_{x}\right)$ which contains $\tilde{B} \mid F_{x}$ (so separates the points of $\left.F_{x}\right)$ and is contained in $\operatorname{cl}\left(\tilde{B} \mid F_{x}\right)$, hence by the StoneWeierstrass theorem $\operatorname{cl}\left(\tilde{B} \mid F_{x}\right)=C\left(F_{x}\right)$. By Lemma 1.3 , (cl $\left.\tilde{B}\right) \mid F_{x}=\operatorname{cl}\left(\tilde{B} \mid F_{x}\right)$. It follows that $\mathrm{cl} \tilde{B}=C(\tilde{X})$, and we conclude that $B=C(X)$ by Bernard's lemma.

COROllary 1.1. Let $A$ be a nontrivial function algebra on a compact Hausdorff space. Let I be a nondegenerate interval and $D$ be a plane domain. Then we have:

(1) Every function in $\operatorname{Op}\left(A_{D}, \operatorname{Re} A\right)$ is harmonic.

(2) Every function in $\mathrm{Op}\left(A_{D}, A\right)$ is analytic [15].

(3) Every function in $\mathrm{Op}\left((\operatorname{Re} A)_{I}, \operatorname{Re} A\right)$ is affine $[11,16]$.

Proof. By Remark 2 we have $\operatorname{Op}^{c}\left(A_{D}, \operatorname{Re} A\right)=\operatorname{Op}\left(A_{D}, \operatorname{Re} A\right), \operatorname{Op}^{c}\left(A_{D}, A\right)$ $=\mathrm{Op}\left(A_{D}, A\right)$, and $\mathrm{Op}^{\prime}\left((\operatorname{Re} A)_{I}, \operatorname{Re} A\right)=\mathrm{Op}\left((\operatorname{Re} A)_{I}, \operatorname{Re} A\right)$. (1) is trivial by Theorem 1. If $h \in \operatorname{Op}\left(A_{D}, A\right)$ then $\operatorname{Re} h, \operatorname{Im} h, \operatorname{Re} z h$, and $\operatorname{Im} z h$ are harmonic by (1), hence $h$ and $z h$ are harmonic, which implies that $h$ is analytic, proving (2). For (3) we may assume $I=[-1,1]$. If $h \in \operatorname{Op}\left((\operatorname{Re} A)_{l}, \operatorname{Re} A\right)$ then $\varphi: z \mapsto h \circ \operatorname{Re} z$ belongs to $\operatorname{Op}\left(A_{U}, \operatorname{Re} A\right)$ where $U=\{z \in C:|z|<1\}$, so $\varphi$ is harmonic by $(1) .0=\Delta(\varphi)=$ $h^{\prime \prime} \circ \operatorname{Re} z$ shows that $h^{\prime \prime}=0$ on $(-1,1)$, hence $h$ is affine, proving (3).

Next we will obtain an analogous result when $A$ is ultraseparating.

Corollary 1.2. Let $A$ be an ultraseparating Banach function algebra on a compact Hausdorff space $X$ and $B$ be a nontrivial Banach function algebra on $X$. Let I be a nondegenerate interval and $D$ be a plane domain. Then we have:

(1) Every function in $\mathrm{Op}^{c}\left(A_{D}, \operatorname{Re} B\right)$ is harmonic.

(2) Every function in $\operatorname{Op}^{c}\left(A_{D}, B\right)$ is analytic or anti-analytic.

(3) Every function in $\mathrm{Op}^{c}\left((\operatorname{Re} A)_{l}, \operatorname{Re} B\right)$ is affine.

Proof. Since $A$ is ultraseparating, condition (*) in Theorem 1 holds. Thus we have (1) by Theorem 1. We will prove (2) in Corollary 2.2. In the same way as in the proof of (3) of Corollary 1.1, we see that (3) holds.

Remark 3. The results (2) and (3) for the case of $B=A$ are obtained in [13 and 12], respectively.

We will show in $\S 2$ that $\operatorname{Op}^{c}\left(A_{D}, B\right) \subset H(D)=\{f: f$ is analytic on $D\}$ or $\mathrm{Op}^{c}\left(A_{D}, B\right) \subset \bar{H}(D)$ for ultraseparating $A$.

2. Functions in $\operatorname{Op}\left(A_{D}, B\right)$. In this section we study functions in $\operatorname{Op}\left(A_{D}, B\right)$ and give a characterization of $\operatorname{Op}^{c}\left(A_{D}, B\right)$ for the case that $B$ is a function algebra and $D=\{z \in C:|z|<1\}$. For a subset $E$ of $C(X)$ we denote by $X / E$ the quotient space of $X$ for $E$, that is, the space defined by identifying the points of $X$ that cannot be separated by $E$.

THEOREM 2. Let $D$ be a plane domain. Suppose that one of the following holds:

(1) There are functions $\varphi_{1}$ and $\varphi_{2}$ in $\operatorname{Op}\left(A_{D}, B\right)$ such that $\varphi_{1}$ is a nonconstant analytic function and $\varphi_{2}$ is a nonconstant anti-analytic function.

(2) There is a function $\varphi$ in $\operatorname{Op}^{c}\left(A_{D}, B\right)$ such that $\varphi$ is neither analytic nor anti-analytic.

Then we have $\operatorname{cl} \tilde{B} \supset \tilde{A},(\tilde{A})^{-}$, where we denote $(\tilde{A})^{-}=\{f \in C(\tilde{X}): \tilde{f} \in \tilde{A}\}$. 
Proof. We first consider the case (1). Without loss of generality we may assume that $\varphi_{1}=\sum_{n=k}^{\infty} a_{n} z^{n}$ and $\varphi_{2}=\sum_{n=m}^{\infty} b_{n} \bar{z}^{n}$ on $\{z \in C:|z|<1\}$ with $a_{k} b_{m} \neq 0$ and that $k, m \geqslant 1$. So there is $1>\gamma>0$ such that

$$
\partial^{k} / \partial z^{k} \varphi_{1}(z) \neq 0 \quad \forall z \in\{z \in C:|z| \leqslant \gamma\}
$$

and

$$
\partial^{m} / \partial \bar{z}^{m} \varphi_{2}(z) \neq 0 \quad \forall z \in\{z \in C:|z| \leqslant \gamma\} .
$$

In the same way as in Lemma 1.2 we have the boundedness principle of $\varphi_{1}$ and $\varphi_{2}$ as follows:

For $j=1$ and 2 there are positive integers $n_{j}$, positive real numbers $\varepsilon_{j}$, functions $F_{j}$ in $\left\{f \in A:\|f\|_{X} \leqslant \gamma / 2\right\}$, and dense subsets $U_{j}$ of $\left\{g \in A: N_{A}\left(g-F_{j}\right)<\varepsilon_{j}\right\}$ with $F_{j} \in U_{j}$, such that $\|g\|_{X} \leqslant \gamma / 2$ and $N_{B}\left(\varphi_{j} \circ g\right)<n_{j}$ for every $g$ in $U_{j}$.

Let $\tilde{f}$ be in $\tilde{A}$. Let $t$ and $\delta>0$ be real numbers with sufficiently small absolute value. By the boundedness principle we have

$$
\varphi_{j}\left(\left(F_{j}\right)+\delta \tilde{f}+t\right) \in \operatorname{cl} \tilde{B} \quad \text { and } \quad \varphi_{j}\left(\left(F_{j}\right)+i \delta \tilde{f}+t\right) \in \operatorname{cl} \tilde{B}
$$

for $j=1$ and 2 . We see that

$$
\left\{\varphi_{j}\left(\left(F_{j}\right)+\delta \tilde{f}+t\right)-\varphi_{j}\left(\left(F_{j}\right)+t\right)\right\} / \delta
$$

and

$$
\left\{\varphi_{j}\left(\left(F_{j}\right)+i \delta \tilde{f}+t\right)-\varphi_{j}\left(\left(F_{j}\right)+t\right)\right\} / \delta
$$

tend to

$$
\operatorname{Re} \tilde{f} \partial / \partial x \varphi_{j}\left(\left(F_{j}\right)+t\right)+\operatorname{Im} \tilde{f} \partial / \partial y \varphi_{j}\left(\left(F_{j}\right)+t\right)
$$

and

$$
\operatorname{Re} i \tilde{f} \partial / \partial x \varphi_{j}\left(\left(F_{j}\right)+t\right)+\operatorname{Im} i \tilde{f} \partial / \partial y \varphi_{j}\left(\left(F_{j}\right)+t\right)
$$

uniformly on $X$ for $j=1$ and 2. Thus we have

$$
\tilde{f} \partial / \partial z \varphi_{1}\left(\left(F_{1}\right)+t\right) \in \operatorname{cl} \tilde{B} \quad \text { and } \quad \tilde{\tilde{f}} \partial / \partial \bar{z} \varphi_{2}\left(\left(F_{2}\right)+t\right) \in \operatorname{cl} \tilde{B} .
$$

In the same way we have

$$
\tilde{f} \partial / \partial z \varphi_{1}\left(\left(F_{1}\right)+i t\right) \in \operatorname{cl} \tilde{B} \text { and } \quad \tilde{\tilde{f}} \partial / \partial \bar{z} \varphi_{2}\left(\left(F_{2}\right)+i t\right) \in \operatorname{cl} \tilde{B} .
$$

In the same way as in the above argument it follows that for a real number $t$ with sufficiently small absolute value we have

$$
\tilde{f} \partial^{2} / \partial z^{2} \varphi_{1}\left(\left(F_{1}\right)+t\right), \tilde{f} \partial^{2} / \partial z^{2} \varphi_{1}\left(\left(F_{1}\right)+i t\right) \in \mathrm{cl} \tilde{B}
$$

and

$$
\overline{\tilde{f}} \partial^{2} / \partial \bar{z}^{2} \varphi_{2}\left(\left(F_{2}\right)+t\right), \overline{\tilde{f}} \partial^{2} / \partial \bar{z}^{2} \varphi_{2}\left(\left(F_{2}\right)+i t\right) \in \operatorname{cl} \tilde{B} .
$$

Continuing in this manner, at the $k$ th and $m$ th stages we get

$$
\tilde{f} \partial^{k} / \partial z^{k} \varphi_{1}\left(\left(F_{1}\right)\right), \tilde{\tilde{f}} \partial^{m} / \partial \bar{z}^{m} \varphi_{2}\left(\left(F_{2}\right)\right) \in \operatorname{cl} \tilde{B}
$$


if we puit $t=0$. Thus we have

$$
|\tilde{f}|^{2} \partial^{k} / \partial z^{k} \varphi_{1}\left(\left(F_{1}\right)\right) \partial^{m} / \partial \bar{z}^{m} \varphi_{2}\left(\left(F_{2}\right)\right) \in \operatorname{cl} \tilde{B} .
$$

By the Stone-Weierstrass theorem we conclude that

$$
\tilde{A},(\tilde{A})^{-} \subset \operatorname{cl} \tilde{B}
$$

since $\left|\left(F_{j}\right)\right| \leqslant \gamma$ on $\tilde{X}$ for $j=1$ and 2 .

Next we consider case (2). We may suppose that there are functions $\varphi^{(1)}$ and $\varphi^{(2)}$ in $\operatorname{Op}\left(A_{U}, B\right)$ for $U=\{z \in C:|z|<1\}$ such that $\varphi^{(1)}$ is continuous on $U$ and not analytic at the origin and $\varphi^{(1)}(0)=0$ and $\varphi^{(2)}$ is continuous on $U$ and not anti-analytic at the origin and $\varphi^{(2)}(0)=0$. We obtain the boundedness principle for $\varphi^{(1)}$ and $\varphi^{(2)}$ as usual:

For each $x$ in $X$, there are positive integers $n^{(1)}$ and $n^{(2)}$, positive numbers $\varepsilon^{(1)}$ and $\varepsilon^{(2)}$, and functions $F^{(1)}$ and $F^{(2)}$ in $\left\{f \in A_{x}:\|f\|_{X} \leqslant \frac{1}{2}\right\}$ which satisfy: for $j=1$ and 2 there is a dense subset $U^{(j)}$ of $\left\{g \in A_{x}: N_{A}\left(g-F^{(j)}\right)<\varepsilon^{(j)}\right\}$ with $F^{(j)} \in U^{(j)}$ such that $\|g\|_{X} \leqslant \frac{1}{2}$ and $N_{B}\left(\varphi^{(j)} \circ g\right)<n^{(j)}$ for every $g$ in $U^{(j)}$.

Let $x$ be a point in $X$. Let $\tilde{x}$ and $\tilde{y}$ be different points in $F_{x} \sim Q\left(A_{x}\right)$. If $\tilde{A}$ separates $\tilde{x}$ and $\tilde{y}$, we see tha $\tilde{A}_{x}$ separates them. For, let $\tilde{f} \in \tilde{A}$ with $0 \neq f(y) \neq f(x)$ and put $\tilde{f}_{x} \in \tilde{A}_{x}$ with $\tilde{f}_{x}(\tilde{y}) \neq 0$. If $\tilde{f}_{x}(\tilde{x})=\tilde{f}_{x}(\tilde{y})$, then $\tilde{f}_{x}(\tilde{y}) \neq \tilde{f}_{x}(\tilde{x})$ and $\tilde{f} \tilde{f}_{x} \in \tilde{A}_{x}$. Thus we may suppose that $F_{x} / \tilde{A}_{x}$ is the quotient space of $F_{x} / \tilde{A}$ which is defined by identifying the points $Q\left(A_{x}\right)$. Let $V=\operatorname{cl} \tilde{B} \cap C(\tilde{X} / \tilde{A})$. By the boundedness principle for $\varphi^{(j)}$ at each $x$ in $X$, we may think of $V$ as a function algebra on $\tilde{X} / \tilde{A}$. We will show that $V=C(\tilde{X} / \tilde{A})$. Since $\operatorname{cl} B=C(X)$ by the theorem of de Leeuw and Katznelson [15], we see that for every $x$ in $X, F_{x} / \tilde{A}$ is a closed restriction set for $V$ and that each antisymmetric set for $V$ is contained in some $F_{x} / \tilde{A}$, as in Lemmas 1.3 and 1.4. So we need only show that $V \mid F_{x} / \tilde{A}=C\left(F_{x} / \tilde{A}\right)$ for every $x$ in $X$. For each $x$ in $X$ put $V_{x}=\left\{\tilde{f}=\left(f_{n}\right) \in V: f_{n}(x)=0 \forall n=1,2, \ldots\right\}$ and let $\left[V_{x}\right]$ denote the uniformly closed algebra generated by $V_{x} \mid F_{x}$ and the constant functions. So we may suppose that $\left[V_{x}\right]$ is a function algebra on $F_{x} / \tilde{A}_{x}$ by the boundedness principle for $\varphi^{(j)}$ for $j=1$ and 2 . Let $p$ denote the point in $F_{x} / \tilde{A}_{x}$ which corresponds to $Q\left(A_{x}\right)$. Let $\tilde{x} \in F_{x} / \tilde{A}_{x} \sim\{p\}$. Since $Q\left(A_{x}\right)=(\{x\} \times N)^{-}$, there is an $\tilde{f}_{2}$ in $\tilde{A}_{x}$ with $\tilde{N}_{A}\left(\tilde{f}_{2}\right) \leqslant \frac{1}{2}$ such that $\tilde{f}_{2}(\tilde{x})=z_{2} \neq 0$. Let $\delta$ be a positive number with $\delta<$ $\min \left\{\varepsilon^{(1)}, \varepsilon^{(2)}\right\} / \tilde{N}_{A}\left(\tilde{f}_{2}\right)$. We see that for every small $\eta>0$, there are $\delta(1)$ and $\delta(2)$ with $0<\delta(j)<\delta$ for $j=1$ and 2 such that $\varphi_{\delta(1)}^{(1)}\left(z, z_{2}\right)$ is not analytic on $\{z \in C$ : $|z|<\eta\}$ and $\varphi_{\delta(2)}^{(2)}\left(z, z_{2}\right)$ is not anti-analytic on $\{z \in C:|z|<\eta\}$. In the same way as in the proof of Theorem 1 we may suppose that

$$
\partial / \partial \bar{z} \varphi_{\delta(1)}^{(1)}\left(0, z_{2}\right) \neq 0 \quad \text { and } \quad \partial / \partial z \varphi_{\delta(2)}^{(2)}\left(0, z_{2}\right) \neq 0 \text {. }
$$

Thus we have positive numbers $\varepsilon^{(3)}$ and $\varepsilon^{(4)}, \varepsilon^{(4)} \leqslant\left|\tilde{f}_{2}(\tilde{x})\right|$, such that

$$
\partial / \partial z \varphi_{\delta(1)}^{(1)}(z, w) \neq 0
$$

for every $(z, w)$ in $\left\{(z, w) \in C^{2}:|z| \leqslant \varepsilon^{(3)}\right.$ and $\left.\left|w-z_{2}\right| \leqslant \varepsilon^{(3)}\right\}$ and

$$
\partial / \partial z \varphi_{\delta(2)}^{(2)}(z, w) \neq 0
$$


for every $(z, w)$ in $\left\{(z, w) \in C^{2}:|z| \leqslant \varepsilon^{(4)}\right.$ and $\left.\left|w-z_{2}\right| \leqslant \varepsilon^{(4)}\right\}$. Define a compact neighborhood $\theta$ of $\tilde{x}$ as follows:

$$
\theta=\left\{\tilde{y} \in F_{x} / \tilde{A}_{x}:\left|\tilde{f}_{2}(\tilde{y})-\tilde{f}_{2}(\tilde{x})\right| \leqslant \min \left\{\varepsilon^{(3)}, \varepsilon^{(4)}\right\} / 2\right\} .
$$

We remark that we may suppose that $\theta$ is a compact subset of $F_{x} / \tilde{A}$. For a function $\tilde{f}$ in $\tilde{A}$ take a complex number $\beta$ with small absolute value, so we have

$$
\varphi^{(j)}\left(\left(F^{(j)}\right)+\beta \widetilde{f f_{2}^{2}}-w \tilde{f}_{2}\right)
$$

is in cl $\tilde{B}_{x}$, so is in $V_{x}$. Thus by Lemma 6 we have

$$
\overline{\tilde{f}} \partial / \partial \bar{z}_{1}\left(\varphi_{\delta(1)}^{(1)}\left(\left(F^{(1)}\right), \tilde{f}_{2}\right) \tilde{\tilde{f}}_{2}^{2}\right) \in V_{x}
$$

and

$$
\tilde{f} \partial / \partial z_{1}\left(\varphi_{\delta(2)}^{(2)}\left(\left(F^{(2)}\right), \tilde{f}_{2}\right) \tilde{f}_{2}^{2}\right) \in V_{x}
$$

Therefore we see that

$$
|\tilde{f}|^{2} \partial / \partial \bar{z}_{1}\left(\varphi_{\delta(1)}^{(1)}\left(0, \tilde{f}_{2}\right) \tilde{\tilde{f}}_{2}^{2}\right) \partial / \partial z_{1}\left(\varphi_{\delta(2)}^{(2)}\left(0, \tilde{f}_{2}\right) \tilde{f}_{2}^{2}\right)\left|F_{x} \in V_{x}\right| F_{x} .
$$

It follows by the Stone-Weierstrass theorem that

$$
C\left(F_{x} / A\right) \times \partial / \partial \bar{z}_{1}\left(\varphi_{\delta(1)}^{(1)}\left(0, \tilde{f}_{2}\right) \tilde{\tilde{f}}_{2}^{2}\right) \partial / \partial z_{1}\left(\varphi_{\delta(2)}^{(2)}\left(0, \tilde{f}_{2}\right) \tilde{f}_{2}^{2}\right) \mid F_{x} / \tilde{A} \subset\left[V_{x}\right] .
$$

Since $\left|\tilde{f}_{2}-z_{2}\right| \leqslant \min \left\{\varepsilon^{(3)}, \varepsilon^{(4)}\right\}$ on $\theta$ we have $C(\theta) \subset\left[V_{x}\right] \mid \theta$. Then we see $C\left(F_{x} / \tilde{A}_{x}\right)=\left[V_{x}\right]$ since $\left[V_{x}\right]$ is a function algebra on $F_{x} / \tilde{A}_{x}$. Because $F_{x} / \tilde{A}$ is a closed restriction set we have $V \mid F_{x} / A=\left[V_{x}\right]+l^{\infty}$. Thus we conclude that

$$
C\left(F_{x} / \tilde{A}\right)=V \mid F_{x} / \tilde{A}
$$

for every $x$ in $X$. So every antisymmetric set for $V$ is a singleton if we view $V$ as a function algebra on $\tilde{X} / \tilde{A}$, hence $V=C(\tilde{X} / \tilde{A})$, that is,

$$
\operatorname{cl} \tilde{B} \supset C(\tilde{X} / \tilde{A}) \supset \tilde{A},(\tilde{A})^{-} .
$$

We prove a generalization of a result of Bernard and Dufresnoy [6] (cf. [13, 14]).

Corollary 2.1. Let $A$ be a Banach function algebra on $X$ and let $D$ be a plane domain. If there is a nonanalytic function on $D$ in $\operatorname{Op}^{c}\left(A_{D}, A\right)$, then we have cl $\tilde{A}=C(\tilde{X} / \tilde{A})$. In particular if $A$ is ultraseparating, then we have $A=C(X)$.

Proof. Put $A=B$. Then we have $\operatorname{cl} \tilde{A}=C(\tilde{X} / \tilde{A})$ by Theorem 2 and the Stone-Weierstrass theorem. If $A$ is ultraseparating, then $\operatorname{cl} \tilde{A}=C(\tilde{X})$. Thus it follows that $A=C(X)$ by Bernard's lemma.

In [2] Bachar asked when $\operatorname{Op}\left(A_{D}, B\right)=H(D)$. We provide information in the case where $A$ is an ultraseparating Banach function algebra or $B$ is a function algebra.

COROLlaRY 2.2. Let $A$ be ultraseparating. Let $D$ be a plane domain. Then one of the following takes place.

(1) $\mathrm{Op}^{c}\left(A_{D}, B\right) \subset H(D)$.

(2) $\operatorname{Op}^{c}\left(A_{D}, B\right) \subset \bar{H}(D)$.

(3) $\mathrm{Op}^{c}\left(A_{D}, B\right)=C(D)$. 
Proof. Suppose that a nonanalytic and nonanti-analytic function $\varphi$ is in $\operatorname{Op}^{\prime}\left(A_{D}, B\right)$ or a nonconstant analytic function $\varphi_{1}$ and a nonconstant anti-analytic function $\varphi_{2}$ are in $\operatorname{Op}^{c}\left(A_{D}, B\right)$. Then by Theorem 2 we have cl $\tilde{B} \supset \tilde{A},(\tilde{A})^{-}$. Since $A$ is ultraseparating, we see that $\operatorname{cl} \tilde{B}=C(\tilde{X})$ by the Stone-Weierstrass theorem. Thus we have $B=C(X)$ by Bernard's lemma. It follows that $\operatorname{Op}^{\prime}\left(A_{D}, B\right)=C(D)$.

In the case where $B$ is a function algebra we can prove the following proposition.

Proposition 1. Suppose that $B$ is a nontrivial function algebra on $X$. Then $\operatorname{Op}^{c}\left(A_{D}, B\right) \subset H(D)$ or Op' $\left(A_{D}, B\right) \subset \bar{H}(D)$ for every plane domain $D$.

Next we characterize $\operatorname{Op}^{c}\left(A_{D}, B\right)$ for a function algebra $B$ on $X$ and for $D=\{z \in C:|z|<1\}$.

Theorem 3. Let $B$ be a function algebra on $X$. Put $D=\{z \in C:|z|<1\}$. Then one of the following takes place.

(1) $\mathrm{Op}^{c}\left(A_{D}, B\right)=H(D)$.

(2) $\mathrm{Op}^{c}\left(A_{D}, B\right)=\bar{H}(D)$.

(3) $\mathrm{Op}^{c}\left(A_{D}, B\right)=C(D)$.

(4) $\mathrm{Op}^{c}\left(A_{D}, B\right)$ is the set of all constant functions on $D$.

In particular, if $A$ is also a function algebra and $X$ is infinite, then we have $\operatorname{Op}^{c}\left(A_{D}, B\right)=\operatorname{Op}\left(A_{D}, B\right)$ by Remark 2. Furthermore we see

(1) happens if and only if $A \subset B \neq C(X)$;

(2) happens if and only if $A \subset \bar{B} \neq C(X)$;

(3) happens if and only if $B=C(X)$.

Proof. By Proposition 1 we see that $\operatorname{Op}^{c}\left(A_{D}, B\right) \subset H(D)$ or $\operatorname{Op}^{c}\left(A_{D}, B\right) \subset \bar{H}(D)$ or $\operatorname{Op}^{c}\left(A_{D}, B\right)=C(D)$. Suppose that constant functions on $\left.D\right\} \varsubsetneqq$ $\operatorname{Op}^{c}\left(A_{D}, B\right) \subset H(D)$. Let $\varphi=\sum_{n=k}^{\infty} a_{n} z^{n}$ be in $\operatorname{Op}^{c}\left(A_{D}, B\right)$. Without loss of generality we may assume that $k \geqslant 1$ and $a_{k} \neq 0$. For every $f$ in $A$ with $f(X) \subset D$, we have $\varphi(f+t)$ and $\varphi(f+i t)$ in $B$ for every real number $t$ with small absolute value. In the same way as in the proof of Theorem 2 we see that

$$
k ! a_{k} f=f \partial^{k} / \partial z^{k} \varphi(0) \in B=f a_{k},
$$

so $f \in B$, for $a_{k} \neq 0$. Thus we conclude that $A \subset B$. It follows that $\operatorname{Op}\left(A_{D}, B\right) \supset$ $H(D)$ since $D=\{z \in C:|z|<1\}$. Suppose that \{constant functions on $D\} \subsetneq$ $\operatorname{Opp}^{\prime}\left(A_{D}, B\right) \subset \bar{H}(D)$. We see that $A \subset \bar{B}$ and $\operatorname{Op}^{\prime}\left(A_{D}, B\right)=\bar{H}(D)$ in the same way as above.

ACKNOWLEDGMENT. The author wishes to express his hearty thanks to the referee for a number of suggestions which have improved the accuracy and readability of the article.

\section{REFERENCES}

1. J. M. Bachar, Jr., Composition mappings between function spaces, Ph.D. Thesis, UCLA, 1970.

2. . Some results on range transformations between function spaces, Proc. Conf. on Banach Algebras and Several Complex Variables, Contemporary Math., vol. 32. Amer. Math. Soc., Providence. R. I.. 1984. pp. 35-62.

3. W. (3. Bade and P. C. Curtis, Jr., Banach algehras on F-spaces, Function Algebras, Proc. Internat. Sympos. on Function Algebras, Tulane University, (F. T. Birtel, ed.). Scott, Foresman \& Co., (Glenview, III. 1966. pp. 9()-92. 
4. B. T. Batikyan and E. A. Gorin, On ultraseparating algebras of continuous functions, Moscow Univ. Math. Bull. 31 (1976). 71-75.

5. A. Bernard, Espaces des parties réelles des éléments d'une algèbre de Banach de fonctions, J. Funct. Anal. 10 (1972), 387-409.

6. A. Bernard and A. Dufresnoy, Calcul symbolique sur la frontière de Šilov de certaines algèbres de fonctions homomorphes, Ann. Inst. Fourier (Grenoble) 25 (1975), 33-43.

7. F. T. Birtel (Editor), Function algebras, Proc. Internat. Sympos. on Function Algebras, Tulane University, Scott, Foresman \& Co., Glenview, Ill., 1966.

8. E. Bishop, A generalization of the Stone-Weierstrass theorem, Pacific J. Math. 11 (1961), 777-783.

9. R. B. Burckel, Characterizations of $C(X)$ among its subalgebras, Marcel Dekker, New York, 1972.

10. P. C. Curtis, Jr., Topics in Banach spaces of continuous functions, Lecture Notes in Aarhus Univ., 1970 .

11. O. Hatori, Functions which operate on the real part of a function algebra, Proc. Amer. Math. Soc. 83 (1981), 565-568.

12. Functions which operate by composition on the real part of a Banach function algebra, Tokyo J. Math. 6 (1983), 423-429.

13. . Functional calculus for certain Banach function algebras, J. Math. Soc. Japan 38 (1986), 103-112.

14. Remarks on the functional calculus on a Banach function algebra (preprint).

15. K. de Leeuw and Y. Katznelson, Functions that operate on non-selfadjoint algebras, J. Analyse Math. 11 (1963), 207-219.

16. S. J. Sidney, Functions which operate on the real part of a uniform algebra, Pacific J. Math. 80 (1979), $265-272$.

17. W. Spraglin, Partial interpolation and the operational calculus in Banach algebras, Ph. D. Thesis, UCLA, 1966.

18. J. Wermer, The space of real parts of a function algebra, Pacific J. Math. 13 (1963), 1423-1426.

Department of Mathematics, School of Education, Waseda University, Nishi-Waseda, SHINJUKU-KU, TOKYO 160, JAPAN

Current address: Department of Mathematics, College of Liberal Arts and Sciences, Kitasato University, 1 Asamizodai Sagamihara City, Kanagawa Prefecture, 228 Japan 